\title{
Playing with Time in Digital Fiction
}

\section{Raine Koskimaa}

University of Jyväskylä, Finland

doi: 10.7358/ijtl-2015-001-kosk

raine.koskimaa@jyu.fi

\section{ABSTRACT}

The exceptional quality of digital fictions lies in their inherently dynamic nature, how they may be flexibly programmed to generate new content and alter the already existing contents. This adds a new temporal level, compared to traditional fictions. Digital games, especially, incorporate aspects of simulation and narration in their structure. As interactive and dynamic media form, games are specifically temporal in nature. They offer us the flexibility and preciseness of digital simulations, with the potential of psychologically engaging narrative qualities, which together open up a whole new field of experimenting with temporally dynamic media. Much of the new media fictions partake in a wider transmedia story worlds with temporal implications of their own. In this article the temporal dimension of the digital fictions the Braid (by Jonathan Blow, 2008) and the Spore (by Will Wright for Maxis, 2008) are discussed. We will focus on how these digital fictions, employing aspects of simulation, play and narration, highlight the idea of time as resource.

Keywords: the Braid (game); digital fiction; fictional time; narration; the Spore (game); simulation; temporality.

\section{INTRODUCTION}

The exceptional quality of digital fictions lies in their inherently dynamic nature, how they may be flexibly programmed to generate new content and alter the already existing contents. This adds a new temporal level, compared to traditional fictions. Already the history of digital fictions presents us with a variety of temporal practices, even though this dimension has not been thoroughly discussed so far.

Digital games, especially, incorporate aspects of simulation and narration in their structure. As interactive and dynamic media form, games are specifically temporal in nature. They offer us the flexibility and preciseness of digital 
simulations, with the potential of psychologically engaging narrative qualities, which together open up a whole new field of experimenting with temporally dynamic media. Much of the digital fictions partake in wider transmedia story worlds. Here, the fictional time necessarily blends with the true time of the audience.

In this article, we will discuss the temporal dimension of two digital fictions, the Braid (by Jonathan Blow, 2008), computer game with time-reversal as game mechanic, and the Spore (by Will Wright for Maxis, 2008), a game with simulated evolutionary time-scale and a wide transmedia dimension.

Games are a specific class of simulations, where time figures also as a game element, posing challenges and limitations to the player. Narratives, according to the Aristotelian tradition, are imitation of human activity. In more modern framework we may consider narratives not only as imitation, but also as simulation of activities. In this article we will focus on digital fictions which employ aspects of simulation, play and narration, and which highlight the temporality of existence and experience.

Digital fiction includes games (with fictional content), digital literature, and transmedia franchises with digital storytelling elements. What is common with all types of digital fictions is that they combine elements of gaming / playing, narrative, and simulation. These aspects are enabled by the fact that by being based on digital media technologies, digital fictions are dynamic, they may be programmed to behave in various ways. The dynamic nature directly relates to the temporal quality of digital fiction, which is more complex than time in more traditional forms of fiction. One of the first attempts to theorize game temporality was Juul (2005), where the play time and the fictional time of the game-world were differentiated, and various mappings between them modeled (141-156). Juul also counted for certain temporal violations which are routinely accepted by players (like the discrepancy between time taken for individual moves in football games as compared to similar moves in real football match, 151-152).

To begin with, it is important to note that time within a fiction is a fictional construct. Thus, it would be possible to play around with temporality and break free from the model of the real world time. Some fundamental properties of time, however, are very seldom violated in fiction. The 'arrow of time', especially, the notion that time only flies in one direction, from past through the present and towards future, is usually maintained. Time-travel stories are a notable exception to this rule, and there are a few fictions where time runs backwards, such as Martin Amis' Time's Arrow (1991), Philip K. Dick's Counter-clock World (1968), and Oldruich Lipskýs film Happy End (1966). In addition to mainly obeying the arrow of time, fictional time is ruled by many conventions, which maintain the temporal realism. This is important 
as time in, especially, literature bears only a vague relation to real time. Gérard Genette, for example, has coined the concept of 'pseudo time' to explain how the amount of text, a spatial measure, is translated into a temporal measure when the amount of text used to describe certain events determines how 'slow' or 'fast' the narration is $(1980,33-35)$. For the purposes of this article, we can define direction, order, and speed as the main aspects of fictional time.

Order and speed are qualities which may be distorted in several ways, but this relates usually to the narration, not to the narrated events. Episodes may be narrated more slowly or quickly (per pseudo time), and they may be narrated non-chronologically as is often the case in murder mystery where the event of finding a dead body is narrated first, and then, piecemeal, the earlier episodes leading to the murder are disclosed. This is different, however, than breaking the causal order in the narrative world.

\section{GAME Time}

We start the scrutiny of temporality in digital fiction from games, as time is a fundamental element in most games (the most notable exceptions being puzzle games and turn-based strategy games) and consequently, it is possible to find a number of ways to manipulate time in them. To begin with, games usually allow going back in time, in the sense of returning to an earlier point within the fictional world of the game.

The going back in time may be installed in different ways. Quite typical is to force the player to return to an initial state of the game, in case of failure in given tasks. If the player fails to avoid the barrels thrown around by Donkey Kong, she is taken back to the beginning and has to start the climbing all over again. It is only in such cases where the initial state is fully resumed that we may consider that as going back in time, whereas if modifications in the player character or in the game environment endure, then it is simply a case of spatial relocation.

Many games enable saving the game state, either at any point or at certain specific places (so-called save points). It is then possible for the player to load the saved state later, in order to re-run the subsequent game from that point on. Loading a saved game is, in effect, a time machine allowing return to earlier states of the fictional game world. In games with narrative content there is often a structure of alternative story lines within the same fictional world, and the player may have several states saved, allowing her to switch between the alternatives. In this case, it is not only a question of travelling in (fictional) time, but also switching between parallel fictional worlds (for example, in Star 
Wars The Knights of the Old Republic, the player may traverse the game both as a Jedi or Sith apprentice). Espen Aarseth has made classification to three temporal levels in games, the event time, the negotiation time, and the progression time. It is specifically the existence of the negotiation time in games, which enables the temporal back-and-forth movement (1999, 37-38).

Even in the most realistic, simulation type of games, the temporal aspect is usually condensed. Whereas in all other aspects realistic perfection is aimed at, the duration of the game is kept unrealistically short. In combat games, so-called 1st Person Shooters, the weapons, for example, are very precisely modelled Still, one round of combat seldom is longer than 5 to 10 minutes. Even though it is understandable from the playability perspective that this sort of adjustment is needed (very few players would be willing, or able, to engage in weeks and months long non-stop campaigns), it is still notable how little this has effect on our appreciation of the realism of the game. Also in sports games, like the FIFA Football Game Series, appraised for their level of realism, you may choose how long the " 45 minutes" of a period actually lasts. The temporal distortions are closely related to the spatial aspects of game worlds, and the player needs, as discussed by Aarseth in relation to The World of Warcraft (2008, 118-119) and Anders Løvlie $(2007,73)$ in relation to the Battlefield 2. According to Juul, the main motivation for these temporal distortions is to avoid what the player's experience as dead time, the need to "perform unchallenging activities for the sake of higher goal" $(2005,155)$ such as traversing a long stretch between two locations.

It is also a stock device in many games that the game actions gradually gain speed. This may be used to make the game harder by increasing the kinesthetic challenge (Karhulahti 2013) as in Tetris, where quicker and quicker reactions are required. The opposite effect is to give the player a 'boost', enabling the player to move faster in the game world and thus making it easier to beat the challenge. Often, the boost effect only lasts for a limited amount of time.

The boosting device already refers to the more general phenomenon of having several temporal layers at play simultaneously. In an arcade racing game collecting a rocket motor makes the player's car move considerably faster as compared to the competing racers, creating a different temporal framework within the game world. Max Payne employs a device called bullet time, which enables the player to slow down action in game world, for a limited amount of time, in order to make it easier to fight the enemies, or, to take certain actions requiring high precision. The player may initiate bullet time at will, but once used, it cannot be initiated immediately again but there is a certain wait period. The bullet time is also mimicked occasionally in short cut scenes in the middle of fights, as some of the enemies killed are stopped mid-air and their falling down is shown in extremely slow motion. 
There are also more complex instantiations of the temporal layering, as in the Civilization game series and many other games belonging to the same genre. In Civilization games the player starts building a town, and, over dozens of turns, creates a civilization with several cities, either fighting the neighboring civilizations or seeking friendly relations with them. Each turn in the game designates a number of years passing by in the fictional game world. During each turn, the player has to micro-manage the daily tasks of her units, make ideological choices of how to develop her civilization and decide which technologies to investigate. There is, then, the layer of the historical development of the civilization, spanning millennia in the fictional world of the game, juxtaposed with daily tasks of the units and city management. Furthermore, the game employs so-called technology-tree, which governs how the technologies may be developed. The technology-tree, as such, is a logical structure telling that one has to develop a wheel first, before a chariot may be built. As it entails causal relationships, however, it imposes a temporal framework of its own upon the fictional game world. The fictional time as counted in fictive years is not always in synchrony with the technology tree, at least if compared to our own history. Certain inventions may appear much earlier or later in the civilization's development as one might expect, and also, especially if the player chooses a strategy in which a particular strand of technology is emphasized, there may co-exist highly developed technology in one field (for example, airplanes) and much less developed ones in other field (rowing boat as the most advanced form of seafaring).

The Civilization games also exemplify the class of turn-based games as opposed to the real-time games. In turn-based games, in a sense, time stands still while the player is making her move and nothing in the game world changes during that pondering period. The actual time spent on playing the game is strictly cut off of the fictional time of the game. In real-time simulation games the opponents continuously proceed in their activities, which puts pressure on the player to make quick decisions, but it also brings along the possibility to simply make mistakes in controlling the game units in haste. In simulation games especially, we can talk about reflective temporality of turnbased simulations and competitive temporality of real-time simulations.

The final aspect to note here is that it is common in games to regard time as a very concrete resource. In game levels where a time limit is set (the level has to be finished within a set time, often shown as a diminishing time bar), the player may win more time, as when collecting a special item stops the counter for a while, or lose it, a failure in certain task either slowing her down or making the counter bar drop instantly. It is this reification and manipulability of time that is very specific to digital games. Timothy Barker has developed the idea how digital technologies produce time, as exemplified in digital art, but there 
are clear corollaries to digital games as well (2012). We may have experiences of time running exceptionally slow, or time flying (when you are having fun, or in a state of flow), but these are subjective experiences and only meaningful in relation to the objective time measured by clocks. In the fictional game worlds, time may really be running faster or slower, and furthermore, we have devices to affect the temporal pace.

\section{CONCEPTUALISATIONS OF Time}

Our notion of time has been characterized in many ways. We refer here to just a few well known examples. Henri Bergson made a distinction between the scientific, measurable time, and the experienced duration. The duration is something qualitative, simultaneously a multiplicity and a unity. It can only be grasped through intuition. When we look at the forms of digital fictions, be it the way how things are presented on screen, or how they are run in the code level, we are dealing with the measurable time. The experienced duration, however, is related to the player on the one hand, and to the fictional characters in as much as they manage to evoke feelings of identification in the player. That is, a game may communicate fictional experiences of duration, even though traditional fiction in literature and film is much better developed in this area. (cf. Bergson 2002)

Historian Fernand Braudel made an influential classification of three temporal levels. The first level, geographical time, is that of the environment, with its slow, almost imperceptible change, with cyclical repetition of recurring ice ages and such. The time scale is millennia. The second level of time comprises social and cultural history. Change at this level is faster than at the level of the environment, but the development of certain inventions from the conception to the integration in the whole society may take hundreds of years, as in the case of printing press (from Gutenberg's invention to the full scale internalization of print text in 1800's). The scale on this level is decades and centuries. The third level is the time of daily events, the history and life-world of individuals. The time scale varies from minutes and hours to days, months and years.

We believe that a fourth level is needed, in addition to Braudel's three, that is, the micro-time of physical events, taking place below or behind the human perception. Much of what happens within the microprosessor takes place on this level. Mega and giga Hertzes speak about this rhythm quite incomprehensible for human brain. Mark N. B. Hansen, for example, has attempted at theorizing this machinic time, connecting it to the "nonconscious neural scale of duration" (235). 
Simulation with digital technologies, offers us a tool to render both micro and macro levels of time to the human event-scale. We may witness the billions of years development of our Cosmos in one hour of a Planetarium show. Or the fragment of a second of a particle collision within a particle accelerator slowed down to minutes. It is exactly this practical aspect of flexible and precise adjusting of the temporal variable which makes simulations so efficient tools for all sorts of practices, and which is behind the temporal multiplicity in many games.

\section{Games AND Multiple Temporalities}

In what follows we'll discuss more in detail two digital games, Jonathan Blow's the Braid, which employs all imaginable temporal distortions integrated in the game play, and Will Wright's (Maxis) the Spore, a game with evolutionary time scale and transmedia extensions. They serve well in exemplifying how most of the digital fictions are based on multiple temporalities (or, multi-temporality following Barker 2012), where some of the temporal frames are dictated by the game system logic, some by the fictional story-content, and still others derive from the player's real-life situation. The set of temporalities is related to the types of the games, and especially the multi-player games bring along their own complexities in the whole (see Tychsen and Hitchens 2007). The multiplication of temporalities may lead to a kind of playful take on time itself, but in many cases one of the temporalities clearly dominates the game experience.

\subsection{The Braid}

The Braid is a game created by Jonathan Blow, originally for Xbox 360 (2008). It won the Independent Games Festival Award in 2006, for 'Innovation in Game Design' (the award was given to an early version of the game, before the commercial publication). The Braid is a platform puzzle, with a framework story provided in text format in transitional passages between the levels, where the player embarks on a journey through a strange world to save a Princess. The main peculiarity of the game is that it enables time-reversal at any point. The player may 'rewind' the events simply by pressing one button. The timereversal is not fully consistent, however, and to proceed in the game, it is often crucial to detect the incoherencies and use them to solve the puzzles.

There are six stages or 'worlds' within the game, each employing a different temporal game mechanic: (the list begins with the World 2, as the World 1 is accessible only after completing each of the other five worlds) 
- World 2: reversal of time by pressing 'Shift'. All actions performed may be reeled back without limitations.

- World 3: specific objects (designated with greenish glow) are not affected by the time reversal. It is possible, for example, to get a key by performing a jump which kills the character. Through time reversal the character is returned to an earlier position and resurrected, but still holding the key which was taken during the fatal action. Moving objects which are needed as jumping platforms can be synchronized by minutely accentuated time reversals, as some of the objects are not affected by the reversal.

- World 4: space and time are intertwined, so that when the character moves to the right the time moves forward, leftward movement triggers reversed time, and if the character stays still or jumps upwards time stays still. This requires extremely careful planning of all actions as it is a highly counterintuitive condition.

- World 5: when the player reverses time, a shadow character appears which performs the actions that the real character took before the reversal. There are such specific objects with which both characters may interact. Some puzzles require coordinated actions of the past and the present character.

- World 6: the character carries a magic ring, which may be used to slow down time within the proximity of it (spatially limited slowing down of time).

- World 1: time runs backwards constantly here and using the time reversal restores the normal movement of objects.

It is obvious that there are close connections to the notions of time and temporality as explained by modern physics here, the time-reversibility being one of the key issues in quantum computing (cf. Brown 2000), time and space entanglement stemming from Einstein's theory of relativity, splitting to parallel realities known through the popular example of Schrödinger's Cat etc. On one level the game can be seen as an instantiation of these kinds of temporal peculiarities in modern physics (cf. Davies 1996).

\subsection{Fictional World vs. Logical System}

There is also a strong narrative aspect in the Braid. In the frame story the gamecharacter Tim tells how he has done something in his life, which he strongly regrets (related to his former girlfriend) and he wishes he would be able to render the deed undone. It is this narrative motivation of the in-game timereversal which makes the Braid so intriguing. It is obvious that the Princess the protagonist is chasing after has at least double meaning. On one level the Princess is the former girlfriend of Tim, but on the metaphorical level the Princess stands for some ultimate goal that Tim the scientist is looking for, truth, 
wisdom, knowledge, power... There are plenty of signs alluding to the possibility that Tim has been involved in developing the first nuclear bombs, the detonation of the first bomb possibly being the terrible deed he wishes to be made undone, and the Princess standing for the mastery over Mother Nature. More elaborated interpretations equal Tim with Albert Einstein, the Princess in this case standing for the Unified Field Theory Einstein did seek for in vain.

The game-play does not relate closely to either of these story lines. There are, however, puzzle pictures within certain game stages, depicting persons who could be related to the love story. The time manipulations on the other hand link to the scientist/Einstein story. Even more challenging would be to reconcile these two stories somehow with each other. What is more important, however, is that there is a recognizable story, evoking a fictional world with characters with which the player may, more or less, identify.

In the beginning of the game the logic of time reversal is quite simple. The possibility of reeling back time is, in principle, similar to the possibility of saving a game state and reloading it after a mistake. The difference is practical, in that the time reversal in the Braid is easier to accomplish, and because of that, enables a kind of real-time trial-and-error method simplifying the task of adjusting certain challenging movements. Of course, it also neatly fits with the frame story and its insistence on undoing past mistakes. In this game level, the only thing that is transferred across the time barrier is knowledge: an earlier game state is reinstantiated but the player possesses the memory of the cancelled events which helps in finding more efficient ways to proceed in the game.

In the subsequent worlds, with elements and objects not affected by the time-reversal we have a more complicated situation. It seems that we are facing an incoherent fictional world, where various elements follow different temporal logic. This is not, however, that strange a situation, and there are several works of literary and film fiction where similar cases can be found. But since we are dealing with a digital game here, there is another dimension involved, too, that is the game as a system of structured challenges. When emphasizing the game aspect of the Braid, it is not necessary to consider the fictive narrative at all, in order to succeed in the game. The logic of controlling the game character and environment can be learned through experimenting with the controls and observing the game behavior without referring to the game narrative. How much an individual player focuses on the fictional narrative, and how much on the game challenges as a formal system, cannot be estimated without empirical research of actual players (which is out of scope of this paper). It is, however, quite apparent that the balance of narrative versus ludic challenge in games with narratives depends both on the qualities of a particular game and the character of each individual player. On this scale, the Braid has invested quite much in integrating the narrative with the game system, but many of the chal- 
lenges are repetitive and kinaesthetic in a way which is prone to alienate the player from the immersion in the fictional world.

There is also a procedural counterpart for the game system in the code level of the Braid. When looking at the digital state machine on which the software code of the game runs, the connection to the fictional world is cut off, and the game system is reduced to a set of logical, rather than temporal, connections. In this framework we are not dealing with any sort of time-paradoxes, but simply with synchronizing different systems of state-machines. This approach is fully based on the measurable (scientific) time of Henri Bergson, of which the personal experience of duration is abolished. Even though we are here stepping to the field requiring empirical research, it might be possible to hypothetically argue that the emphasis on measurable time happening during so-called grinding periods in game play (extended periods of highly repetitive and mechanical action), partly explain the often reported failure to notice the amount of time spent on playing digital games.

The Braid seems to confirm the notions of the elusive nature of time. Even though it is probably the richest example of time-based game mechanics, when each of the mechanics is looked closely enough, they seem to lose their temporal nature in favour of logical and spatial puzzles. What is most intriguing, after all, in the Braid, is its playful approach towards the mystery of time. It does provide ingredients for deep philosophical reflection for those so inclined, but one doesn't have to delve in such reflections in order to enjoy the wild experimenting with the game world.

\subsection{The Spore - Playing with Evolution}

One of the most ambitious games released lately has been the game called the Spore, which is a simulation of long-term processes of evolution within the game world. Even though Spore, as an online game, has a much longer time span than usual stand-alone games, it still needs to condense the temporal dimension considerably. It would be worth examining more in detail, how the evolutionary processes develop within this simulated world. In our real world evolution requires a huge amount of selection situations, for certain tendencies to emerge. Artificial, designed factors need to be introduced in the game world, to facilitate faster evolution.

The Spore allows a player to control the development of a species from its beginnings as a microscopic organism to an intelligent and social creature, eventually able to interstellar exploration as a spacefaring culture. The evolutionary scope of the game is combined with open-ended gameplay based on procedural generation allowing emergent phenomena. Whereas the game in 
stand-alone mode is limited to relatively short play time and offers closure through alternative winning states, it is possible to transport one's creatures to the online Sporepedia, and that way, to other players' Spore Worlds where it can continue its life independent of its creator.

There is a strong element of transmediality in the Spore. In the game, there is a built-in functionality to release one's own video captures in the Youtube Spore Channel. The Sporepedia is a forum, where the players may upload their creations done with Spore editors, and share them with other players. The Sporepedia keeps track of every creature and provides an alternative mode for the Spore experience, where the game itself turns into a complex tool for content creation and communication device with the rest of the community. There is also a number of the Spore creatures mimicking cartoon, game, and other media characters, likening it to fan fiction in certain aspects. An in-game SporeStore is also available, where it is possible to purchase Spore-based merchandise like t-shirts, posters, and expansion packs.

Temporally the Spore provides an evolutionary time-scale, with an even longer span than the civilization building games (like Sid Meier's Civilization Series), as the game begins from the early stages of life on earth including Cell and Creature stages. At the other end, there is the ultimate goal of the Space stage, as in most civilization games. The Spore, thus covers billions of years in life's evolution, which requires speeding up of the game time considerably. There are also significant temporal lapses in that the game entities abruptly jump from one stage to another, for example from Cell Stage to Creature Stage, with undefined period skipped in between. The various stages in the game are also unevenly divided, the cell stage covering billions of years, whereas the civilization stage is a matter of millennia. Thus, the speeding up is several factors higher in the early stages compared to later stages.

The sense of temporal direction, the arrow of time, is provided by the development of the game entities. The player has a god-like power to affect this development through various selections, but the backbone of evolution is enabled by the "procedural generation" based on a programmed behavior in each creature. This, in a sense, should enable emergent phenomena as the creatures react to the changing environment, but in practice, the game programming severely limits the development only allowing certain "evolutionary steps". The evolutionary progression only proceeds until certain end states, after which the creatures may still continue their lives in the player's own, or in other players', Spore Worlds, but it does not evolve any more. This juxtaposes two modes of temporality, one seeing time in teleologic terms, as progression towards ever higher states of being, the other being fundamentally circular, in that when there is no progression but only various permutations of possible states this ultimately leads to every state recurring endlessly. 
In addition to the grand time scale, the Spore is also interesting in the way how it plays along parallel temporalities. When a creature is transferred to another player's Spore world, it shifts from one temporal continuum to another one. Following one's own creatures in parallel worlds happens through the Sporepedia. This is quite different activity compared to engagement with a game. The Sporepedia-mediated experience in many ways is close to participating in social media, with frequent but usually short peeks to how things are evolving in the Spore universe. The combination of deep engagement with game world and the frequent visits to the social sphere is what characterizes the transmedia experience more in general.

\subsection{Time in Transmedia}

The transmedia worlds usually refer to such fictional universes as based on the Matrix or Star Wars franchises which deliver their stories over various media (films, games, tv series, books, mobile content etc.) the Spore is not the most typical example of transmedia, but the way how the players' participatory activity is central for developing new content to the Sporepedia and how the game world expands to the realm of additional products and merchandises is very much transmedial.

Temporally, the most notable aspects of transmedia is the challenge in maintaining coherence in distributed story worlds, and the intertwining of fictional time with the player's real time. Because of the distributed nature of transmedia storyworlds and storytelling, the producers usually develop and refer to so-called Transmedia Production Bible which provides the canon of the fictional world. Each individual episode, in every format possible, has to follow the Production Bible, to avoid contradictions and confusions. Carlos Scolari has listed four strategies (found in the TV series 24) to expand the main storyline: the creation of interstitial microstories, parallel stories, peripheral stories, and, user-generated content platforms and fanfiction $(2009,598)$. The interstitial stories fill in temporal gaps between episodes, thus making the flow more continuous. Parallel stories expand the story world inside the given temporal frame, whereas peripheral stories may stay more ambiguous in respect to the temporal continuum as they are not affecting the central characters or events. Users are not tied to the Production Bible, and the usercreated content may contradict with the main story line. In some cases, this is an intentional purpose, as in much of the so-called slash fiction where alternative (sexual) identities and orientations are created for the fictional characters. Often different additional modalities are presented though different media, like interstitial micro stories as webisodes, and peripheral stories as print 
novels, which means that also the way how the users engage with them differ. There are then various modes to suit the circumstantial needs of the user, which enables the user to access the fictional world more flexibly, this being the main reason that the experience is increasingly seamlessly fusing with the everyday experience.

There is not much research on the consumption patterns in relation to transmedia storytelling, and the time distribution between various transmedia elements, but in regards to game like Spore we may assume, that the everyday measurable time becomes more dominant in perceiving the development of one's creature, when it is followed through the Sporepedia, rather than the in-game fictional time scale. It is also quite possible, that the time spent in a state of flow inside game world affects our sense of duration also in physical reality. According to Hansen, that might be understood as "enlarging of the now of perceptual consciousness" $(2004,257)$.

\section{Competing Times}

The peculiarity of digital fictions lies in their dynamic nature. The dynamics, in its turn, is based on computer code executed by microprocessors. As computing devices have evolved into an expressive media (or metamedia, as it is also argued) the digital fictions may combine the flexibility and preciseness of digital simulations with the potential of psychologically engaging media narratives. This in itself opens up a new field of experimenting with temporally dynamic media contents, but also changes our habitual ways to engage with media.

The Braid foregrounds our implicit notions of the flow and direction of time, in making the game time reversible, but also manipulatable in various other ways. In a deeper level it raises ethical questions implied in the situation where it would be possible to return to previous points in time and changes one's choices - would that mean that we are obliged to erase certain deeds with negative catastrophic consequences, even if that would also annihilate most of our life and its accomplishments? As a game, however, it emphasizes the competitive temporality to the extent, that there may not be enough of reflection time for the ethical questions to be evoked, in the first place.

In the Spore, the temporal interest lies foremost in the huge, evolutionary time scale of the game, which simulates the development of living organisms from simple cell stage to space faring civilization. This requires both condensing time within the stages, and leaving wide gaps between the stages. Whereas the game time has predetermined, definite endings, the creatures may continue their life in other players' Spore worlds though the Sporepedia 
website. In a transmedial way the user generated content, like the videos made using the Spore creatures, may provide interstitial stories showing what has happened during the transitional gaps between the stages, while the creatures in parallel Spore worlds may be seen as parallel or peripheral stories. The fictional time is intertwined with the player's real time more intimately when it is possible to log in to the Spore events in various modes, not just through engaged game play.

The examples discussed here seem to confirm the notion that in playing the real time seems to be dominating over the fictional time. It does not entail, however, that the fictional time would be useless, but rather, that the players are quite flexible towards the fictional time. It can be tweaked in many ways, as long as certain key moments are maintaining their relative places in the temporal continuum. When this kind of flexible notion of time is transferred back to the real life experience, it may result in the "timeless time" of the information networks, as Manuel Castells has described it. As there is blending of several temporal modalities taking place, it might be more accurate, however, to call it rather multiple time than a timeless one. When time is considered as a resource, the more acquainted one gets to the multiple time through games and other digital fictions, the better she is able to manage that valuable resource.

\section{REFERENCES}

Aarseth, Espen. 1999. "Aporia and Epiphany in Doom and The Speaking Clock: The Temporality of Ergodic Art", in Cyberspace Textuality. Computer Technology and Literary Theory, edited by Marie-Laure Ryan, 31-41. Bloomington and Indianapolis: Indiana University Press.

Aarseth, Espen. 2008. "A Hollow World: World of Warcraft as Spatial Practice", in Digital Culture, Play, and Identity. A World of Warcraft $®$ Reader, edited by Hilde G. Corneliussen and Walker Jill Rettberg, 111-122. Cambridge, MA: The MIT Press.

Barker, Timothy. 2012. Time and the Digital. Connecting Technology, Aesthetics, and a Process Philosophy of Time. Hanover, NH: Darthmouth College Press.

Bergson, Henri. 2002. Key Writings. London, England \& New York, NY: Continuum.

Brown, Julian. 2000. Minds, Machines and the Multiverse. The Quest for the Quantum Computer. New York, NY: Simon \& Schuster.

Davis, Paul. 1996. About Time. Einstein's Unfinished Revolution. New York, NY: Simon \& Schuster.

Genette, Gérard. 1980. Narrative Discourse. An Essay in Method. Ithaca, NY: Cornell University Press. 
Hansen, Mark B.N. 2004. New Philosophy for New Media. Cambridge, MA: The MIT Press.

Juul, Jesper. 2005. Half-real. Video Games between Real Rules and Fictional Worlds. Cambridge, MA: The MIT Press.

Karhulahti, Veli-Matti. 2013. "A Kinesthetic Theory of Videogames: Time-Critical Challenge and Aporetic Rhematic." Game Studies 13 (1). Last access December 22, 2014. URL: http:/gamestudies.org/1301/articles/ karhulahti_kinesthetic_theory_of_the_videogame.

Løvlie, Anders Sundnes. 2009. The Rhetoric of Persuasive Games: Freedom and Discipline in America's Army. Saarbrücken: VDM Verlag.

Scolari, Carlos. 2009. "Transmedia Storytelling: Implicit Consumers, Narrative Worlds, and Branding in Contemporary Media Production." International Journal of Communication 3: 586-606.

Tychsen, Anders, and Michael Hitchens. 2007. "Interesting Times. Modeling Time in Multi-Player and Massively Multi-Player Role Playing Games", in Proceedings of The 7th International Digital Arts and Culture Conference. The Future of Digital Media Culture, edited by Andrew Hutchison, 375-384. Perth: Curtin University of Technology. 